\title{
Arquitetura da informação para ambientes informacionais digitais: integração de serviços de personalização e customização
}

\author{
Information architecture for digital informational environments: \\ integration of personalization and customization services
}

\author{
Liriane Soares de Araújo de CAMARgo (1) e Silvana Aparecida Borsetti Gregorio VIDOTTI (2) \\ Unesp, Av.Hygino Muzzi Filho, 737, Caixa Postal:181, CEP: 17.525.900, Marília-SP, Brasil, liria- \\ ne@marilia.unesp.br. (2) vidotti@marilia.unesp.br
}

\begin{abstract}
Resumen
La Arquitectura de la Información (AI) auxilia el desarrollo de ambientes informacionales digitales buscando contribuir a la interacción usuario-sistema. Se presentan los servicios de personalización y customización como módulos integrantes de una arquitectura de información para ambientes informacionales digitales. La propuesta de integración consiste en presentarlos en una estructura única, como un catálogo de tipos de servicios. Esa estructura consiste en una tabla que contiene un nombre para el servicio y la descripción del mismo, así como observaciones sobre público objetivo y directrices de implantación.
\end{abstract}

Palabras clave: Arquitectura de la Información. Personalización. Ambientes informacionales digitales.

\section{Introdução}

A Arquitetura da Informação é uma área que está se consolidando na Ciência da Informação, objetivando facilitar os processos de organização, recuperação, representação e navegação da informação, seja na interface, na estrutura do ambiente ou no conteúdo do objeto digital. Esses processos devem considerar o usuário a fim de satisfazer suas necessidades informacionais. Para isso, são necessários estudos de usuários para elaboração de serviços que facilitem a navegação, filtrando e direcionando informações para grupos de usuários específicos.

Assim, serviços de personalização e customização podem ser citados como exemplos de atividades que podem aumentar a usabilidade, oferencendo um ambiente mais individualizado e específico. Baseado nesse contexto, a proposta deste artigo consiste em integrar esses tipos de serviços a uma arquitetura da informação para complementar os serviços desenvolvidos e disponibilizados em um ambiente informacional digital.

\begin{abstract}
Information Architecture (IA) aids in development of digital information environments aiming at facilitating the user-system interaction. Personalization and customization services are presented as modules of information architecture for digital information environments. The proposal is presenting them in a unique structure, as a catalogue of service types. This architecture consists in a table which contains a name for the service and a description of it, as well as observations about the target-public and implantation guidelines.
\end{abstract}

Keywords: Information Architecture. Personalization. Customization. Digital information environments.

Os serviços de personalização e customização possibilitam alterações de componentes visuais e informacionais na interface, bem como reconhecimento de usuários de forma individual e recomendação de informações específicas. Algumas atividades são consideradas tanto de personalização quanto de customização, porém existem diferenças entre elas, as quais são apresentadas por autores da área como: Morville e Rosenfeld (2006, p. 139, tradução nossa) que relatam que "personalização envolve serviços de páginas inter-relacionadas para usuários baseados em um modelo de comportamento, necessidades ou preferências daquele indivíduo". Já customização "oferece ao usuário o controle direto sobre a combinação de apresentação, navegação, e opções de conteúdos".

Baseada nesses serviços, a proposta consiste em apresentar um conjunto de atividades de personalização e customização em uma estrutura, contendo nome e descrição de cada atividade com o intuito de auxiliar desenvolvedores na escolha e implantação das mesmas. 
A identificação dessas atividades ocorreu em duas etapas: primeiramente foi realizado um levantamento literário e depois uma análise em vários tipos de websites.

Espera-se como resultados que tal integração facilite a recuperação e uso da informação, aumentando a usabilidade do ambiente informacional digital.

\section{Arquitetura da informação digital}

Segundo Rosenfeld e Morville (1998) a Al é focada em trazer para o contexto digital, os princípios de design e arquitetura, podendo assim projetar como componentes visuais $\mathrm{e}$ informacionais podem ser modificados e como informações personalizadas serão processadas, combinadas e recomendadas.

Pode-se afirmar que a $\mathrm{Al}$ aborda interface e conteúdo, contudo ela também envolve contexto e usuário. Nesse sentido, Brancheau e Wetherbe (1986 apud Lima-Marques e Macedo, 2006, p. 248) adotam o conceito de Al como "uma metodologia para estruturação de sistemas de informação aplicada a qualquer ambiente informacional, sendo este compreendido como o espaço que integra contexto, conteúdos e usuários".

Tosete Herranz e Rodríguez Mateos (2004) relatam que $\mathrm{Al}$ é tanto uma arte como uma ciência centrada na gestão da informação e no projeto de website, cuja função primordial é facilitar aos usuários o acesso e a recuperação da informação.

Assim, pode-se relatar que a Al não se restringe apenas ao desenvolvimento de ambientes informacionais (beneficiando apenas o desenvolvedor), ela também aborda o usuário, facilitando a utilização e aumentando a usabilidade do ambiente informacional, incentivando a utilização de recursos interativos para melhorar a comunicação e produção de informações.

A Al pode auxiliar na projetação de serviços disponibilizados em um ambiente informacional digital. Entre eles pode-se citar: serviços de busca, de navegação, de interoperabilidade, de segurança, de personalização entre outros. Além desses serviços a Al pode auxiliar na utilização de princípios de usabilidade, ergonomia, acessibilidade, design de interação, qualidade etc.

Entre esses serviços e princípios, este artigo enfoca a personalziação e a customização, os quais serão discutidos na próxima seção.

\section{Personalização e customização}

Schilke et al. (2004, p. 381, tradução nossa) enfatizam que "personalização não deve ser confundida com customização", pois a customização geralmente lida com a aparência do site (por exemplo cores e fontes) e a personalização geralmente lida com as preferências dos usuários (por exemplo sugestões de informações de interesse do usuário).

Baseado em Mobasher et al. (2001), neste artigo é considerado que a personalização envolve atividades de filtragem, processamento e direcionamento de informações específicas e que a customização envolve atividades de modificação aos componentes visuais e informacionais da interface para e pelos usuários.

Em resumo, com a personalização supõem-se o quê o usuário quer e com a customização o usuário diz ao sistema o que ele quer. "A diferença entre customização e personalização está relacionada com o nível de participação dos usuários na adaptação realizada no ambiente digital" (Vieira, 2005, p. 29). Por exemplo: um site que permite o usuário escolher o papel de parede da página está oferecendo um serviço de customização. Já uma livraria virtual que oferece um livro baseado na percepção que tem a respeito do usuário está oferecendo um serviço de personalização (Vieira, 2005).

Muitos autores denominam para personalização o termo "sistema de recomendação", pois são definidos como "um tipo particular de personalização, que aprendem sobre as necessidades de uma pessoa e então proativamente identificam e recomendam informações que satisfaçam tais necessidades (Callan, et al., 2003, p. 2, tradução nossa) ".

Complementar a essa definição Herlocher et al. (2004, p. 5-6, tradução nossa) afirmam que esses sistemas "usam a opinião de comunidades de usuários para ajudar indivíduos desta comunidade de forma mais efetiva e identificar conteúdos de interesses dentre um conjunto de escolhas potenciais".

Considerando que para a utilização do serviço de personalização é necessário coletar dados dos usuários, autores como Callan et al. (2003) e Dias (2003) comentam sobre alguns exemplos de dados que podem ser coletados como: habilidades cognitivas, diferenças individuais, padrões de comportamento individual ou grupal, domínio de assuntos, tarefas e ambientes de trabalho, papel ou função específica, familiaridade com computadores, nível de conhecimento do domínio da aplicação, freqüência de uso da aplicação, contexto sócio-cultural, etc. 
Baseado em todo o contexto exposto, pode-se afirmar que o sucesso das aplicações de personalização depende do conhecimento acerca dos consumidores como preferências pessoais e comportamentos armazenados sob a forma de perfis. Após a disponibilização de informações personalizadas, a satisfação dos usuários podem ser medidas, e as respostas dos consumidores podem ser usadas para ajustar as estratégias de personalização em um ciclo de feedback iterativo (Adomavicius e Tuzhilin, 2005).

\section{Integração de serviços de personalização e customização em arquitetura da informação}

A seguir apresentam-se alguns projetos publicados por autores da área em artigos científicos:

Cosinger (1994-95 apud Jeevan e Padhi, 2006) descreve um modelo de necessidades de usuários para apoiar forma e conteúdo por meio de uma abordagem de aquisição, representação e exploração de modelos de usuário. Cheong et al. (2002 apud Jeevan e Padhi, 2006) descreve a implementação de um sistema de gestão colaborativo de conteúdos multimída para organizar, integrar e compor cursos interativos e personalizados. Chen e Chen (2007) propõem um sistema de recomendação para uma biblioteca digital. Cardoso e Oliveira (2000) apresentam a proposta da i-LIB, que é um protótipo de interface de biblioteca digital configurável ao perfil do usuário, de modo a estimular o descobrimento e utilização de acervos digitais. Walter et al. (2007, p. 73 ) apresentam um modelo para sistema de recomendação baseado em confiança, que combina conceitos de redes sociais e seus relacionamentos. Eirinaki et al. (2004, p. 72 , tradução nossa) introduzem um método de recomendação, o qual integra dados de uso registrados em logs da web, e os relacionamentos conceituais entre os documentos da web.

Baseado nos projetos apresentados pode-se afirmar que muitas iniciativas sobre personalização e customização estão sendo desenvolvidas e muitos pesquisadores acreditam que esse serviço será muito utilizado pelos ambientes e usuários. Contudo, pode-se perceber que em cada projeto, esses serviços (em específico personalização) possuem abordagens diferentes, constituindo atividades diferentes.

Sendo assim, apresenta-se a seguir um quadro (Quadro I) que mostra as diversas atividades que podem ser realizadas em um processo de personalização e customização.

\begin{tabular}{|c|c|c|}
\hline Nome & Descrição & Observação \\
\hline $\begin{array}{l}\text { Recomendaç } \\
\text { ão de } \\
\text { informações }\end{array}$ & $\begin{array}{l}\text { Recomenda-se } \\
\text { produtos, } \\
\text { documentos, } \\
\text { imagens, sons, } \\
\text { notícias, índice, } \\
\text { ferramentas de } \\
\text { busca, comunidades } \\
\text { de usuários etc., de } \\
\text { acordo com dados do } \\
\text { usuário. }\end{array}$ & $\begin{array}{l}\text { Os dados dos } \\
\text { usuários podem ser } \\
\text { coletados de forma } \\
\text { explícita ou implícita. } \\
\text { Algumas técnicas } \\
\text { que podem ser } \\
\text { utilizadas são: } \\
\text { filtragem } \\
\text { colaborativa, } \\
\text { filtragem baseada em } \\
\text { conteúdo e frames de } \\
\text { recomendação. }\end{array}$ \\
\hline $\begin{array}{l}\text { Alteração de } \\
\text { estrutura e } \\
\text { conteúdo }\end{array}$ & $\begin{array}{l}\text { O usuário pode } \\
\text { reorganizar objetos } \\
\text { no ambiente, alterar } \\
\text { componentes visuais } \\
\text { e informacionais na } \\
\text { interface. }\end{array}$ & $\begin{array}{l}\text { A técnica utilizada } \\
\text { para essa atividade } \\
\text { depende do } \\
\text { desenvolvedor, que } \\
\text { oferece opções de } \\
\text { configuração ao } \\
\text { usuário. }\end{array}$ \\
\hline $\begin{array}{l}\text { Colabora-ção } \\
\text { de conteúdo } \\
\text { e de } \\
\text { representa- } \\
\text { ção de } \\
\text { documento }\end{array}$ & $\begin{array}{l}\text { Vários usuários } \\
\text { podem elaborar um } \\
\text { mesmo documento } \\
\text { ou representar } \\
\text { documentos. }\end{array}$ & $\begin{array}{l}\text { Um exemplo dessa } \\
\text { atividade pode ser } \\
\text { vista no google docs } \\
\text { (http://www.google.co } \\
\text { m/google-d-s/intl/pt- } \\
\text { BR/tour1.html) que } \\
\text { possibilita que vários } \\
\text { usuários elaborem } \\
\text { um mesmo } \\
\text { documento. }\end{array}$ \\
\hline $\begin{array}{l}\text { Transcrição } \\
\text { de mídias }\end{array}$ & $\begin{array}{l}\text { O sistema pode } \\
\text { transcrever textos } \\
\text { para áudio ou por } \\
\text { uma transcrição em } \\
\text { Língua de sinais ou } \\
\text { vice-versa. }\end{array}$ & $\begin{array}{l}\text { Para essa atividade, } \\
\text { o desenvolvedor } \\
\text { deverá analisar as } \\
\text { tecnologias } \\
\text { existentes e mais } \\
\text { apropriadas. }\end{array}$ \\
\hline $\begin{array}{l}\text { Ranking ou } \\
\text { classifica-ção } \\
\text { de opções de } \\
\text { busca }\end{array}$ & $\begin{array}{l}\text { O sistema realiza um } \\
\text { ranking ou uma } \\
\text { classificação de } \\
\text { opções baseadas na } \\
\text { importância do } \\
\text { assunto, termo de } \\
\text { busca, tesauro, } \\
\text { ontologias ou outras } \\
\text { informações } \\
\text { relevantes para essa } \\
\text { ordem de priorização. }\end{array}$ & $\begin{array}{l}\text { A ordem de } \\
\text { priorização pode ser } \\
\text { por: número de } \\
\text { acesso, número de } \\
\text { citações, número de } \\
\text { palavras-chaves, etc. }\end{array}$ \\
\hline $\begin{array}{l}\text { Consulta } \\
\text { personaliza- } \\
\text { da }\end{array}$ & $\begin{array}{l}\text { O sistema oferece } \\
\text { um ambiente em que } \\
\text { o usuário possa filtrar } \\
\text { informações. }\end{array}$ & $\begin{array}{l}\text { Geralmente, essa } \\
\text { atividade é realizada } \\
\text { em ferramentas de } \\
\text { busca avançada } \\
\text { utilizando várias } \\
\text { estratégias de busca. }\end{array}$ \\
\hline $\begin{array}{l}\text { Compartilha } \\
\text { mento de } \\
\text { interesses } \\
\text { em comum }\end{array}$ & $\begin{array}{l}\text { O sistema oferece } \\
\text { um local de } \\
\text { discussão. }\end{array}$ & $\begin{array}{l}\text { Essa atividade pode } \\
\text { ser vista em vários } \\
\text { ambiente como o } \\
\text { messenger } \\
\text { (http://download.live.c } \\
\text { om/messenger). }\end{array}$ \\
\hline
\end{tabular}

Quadro I. Atividades de personalização e customização

Além dessas atividade, foram identificadas outras atividades por meio de análises em vários tipos de websites como Amazon (www.amazon.com), Submarino (www.subma rino.com.br), Google (www.google.com), Youtube (www.youtube.com) e Orkut (www.orkut. 
com). As atividades de customização identificadas estão apresentadas no Quadro II. E as atividades de personalização no Quadro III.

\begin{tabular}{ll}
\hline Nome & Descrição \\
\hline $\begin{array}{l}\text { Escolha de } \\
\text { idiomas }\end{array}$ & $\begin{array}{l}\text { Possibilita ao usuário escolher um idioma } \\
\text { de sua preferência. }\end{array}$ \\
\hline $\begin{array}{l}\text { Ordenação da } \\
\text { busca }\end{array}$ & $\begin{array}{l}\text { Possibilita ordenar as 'informações' por } \\
\text { várias categorias como: data, ordem } \\
\text { alfabética, preço, os mais vendidos, } \\
\text { região etc. }\end{array}$ \\
\hline $\begin{array}{l}\text { Visualização } \\
\text { dos itens } \\
\text { selecionados }\end{array}$ & $\begin{array}{l}\text { Possibilita ao usuário visualizar apenas } \\
\text { os itens selecionados. }\end{array}$ \\
\hline $\begin{array}{l}\text { Indicação de } \\
\text { quantidade de } \\
\text { resultados por } \\
\text { página }\end{array}$ & $\begin{array}{l}\text { Possibilita ao usuário escolher a } \\
\text { página. }\end{array}$ \\
\hline $\begin{array}{l}\text { Opção de } \\
\text { formatos de } \\
\text { informações }\end{array}$ & $\begin{array}{l}\text { Possibilita ao usuário escolher um } \\
\text { formato para ser apresentado na página, } \\
\text { representando um documento ou uma } \\
\text { busca efetuada. }\end{array}$ \\
\hline $\begin{array}{l}\text { Inserção de } \\
\text { informações }\end{array}$ & $\begin{array}{l}\text { Possibilita que o usuário customize seu } \\
\text { perfil com mensagens. }\end{array}$ \\
\hline
\end{tabular}

Quadro II. Atividades de customização

\begin{tabular}{|c|c|}
\hline Nome & Descrição \\
\hline $\begin{array}{l}\text { Espaço de } \\
\text { sugestão }\end{array}$ & $\begin{array}{l}\text { Possibilita um espaço (caixa de texto) } \\
\text { para que o usuário opine sobre o } \\
\text { ambiente. }\end{array}$ \\
\hline $\begin{array}{l}\text { Recebimento } \\
\text { de informações } \\
\text { por e-mail de } \\
\text { interesses } \\
\text { pessoais }\end{array}$ & $\begin{array}{l}\text { Possibilita várias opções ao usuário para } \\
\text { que ele possa escolher as informações } \\
\text { que ele deseja receber por seu e-mail. }\end{array}$ \\
\hline $\begin{array}{l}\text { Relaciona- } \\
\text { mento com as } \\
\text { informações } \\
\text { procuradas }\end{array}$ & $\begin{array}{l}\text { Oferece ao usuário outras opções de } \\
\text { busca relacionadas ao termo pesquisado. }\end{array}$ \\
\hline $\begin{array}{l}\text { Opção "indicar } \\
\text { para um amigo" }\end{array}$ & $\begin{array}{l}\text { Possibilita indicar o ambiente ou alguma } \\
\text { 'informação' para um amigo. }\end{array}$ \\
\hline $\begin{array}{l}\text { Determinação } \\
\text { de prioridades } \\
\text { e preferências }\end{array}$ & $\begin{array}{l}\text { Possibilita ao usuário determinar qual } \\
\text { 'informação' é mais relevante para ele. } \\
\text { Pode ser aplicada para organizar buscas, } \\
\text { disponibilizar informações na página } \\
\text { principal do usuário, localizar algum } \\
\text { produto, entre outras situações. }\end{array}$ \\
\hline
\end{tabular}

Quadro III. Atividades de personalização

Vale ressaltar que outros tipos de alteração de componentes visuais e de informações também fazem parte das atividades de customização e que essas atividades podem ser combinadas para a otimização dos serviços.

\section{Considerações finais}

Os serviços apresentados requerem estudos de usuários, podendo utilizar bases de perfis. Os dados dos usuários podem ser de vários tipos como pessoais, profissionais, de conteúdo, comportamentais, de utilização e avaliação do sistema.

Os serviços de personalização e customização são diferenciados pelo fato de que o primeiro tipo de serviço utiliza informações de usuários, porém não proporciona ao mesmo controle e interatividade por meio de modificações visuais e informacionais, ao contrário da customização, que não utiliza base de perfis, mas oferece flexibilidade para tais alterações. O serviço de personalização também é considerado como sistema de recomendação, que sugere e direciona informações específicas para usuários específicos.

Algumas atividades identificadas foram: recomendar informações, elaborar documentos de forma colaborativa, transcrever mídias, classificar buscas, oferecer busca personalizada, relacionar informações e determinar prioridades, além de alterar estrutura e conteúdo do ambiente, possibilitar inserção de novas informações, escolher idioma, ordenar buscas, escolher formatos etc.

Pode-se afirmar que existe uma forte tendência em utilizar serviços interativos e personalizados para auxiliar na aquisição, produção e uso da informação e do conhecimento.

Espera-se que a apresentação das atividades de personalização e customização possa contribuir para a utilização das mesmas em ambientes informacionais digitais.

Para estudos futuros pode-se implantar e testar serviços de personalização e customização identificando os mais apropriados de acordo com público-alvo e tipo de ambiente.

\section{Referências}

Adomavicius, G.; Tuzhilin, A. (2005). Personalization Technologies: a process-oriented perspective. // Communications of the ACM. 48:10 (2005) 83-90. http://portal.acm.org/ft gateway.cfm?id=1089109\&type= pdf\&coll=Portal\&dl=ACM\&CFID=29687628\&CFTOKEN= 12483935 (mai. 2008).

Callan, J.; Smeaton, A.; Beaulieu, M.; Borlund, P.; Brusilovsky, P.; Chalmemrs, M.; Lynch, C.; Riedl, J.; Smyth, B.; Straccia, U.; Toms, E. (2003). Personalisation and Recommender Systems in Digital Libraries. // Joint NSFEU DELOS. Working Group Report (2003). http://www.dli2.nsf.gov/internationalprojects/working_gro up_reports/personalisation.doc (20 mar. 2008).

Chen, C.-C.; Chen, A.-P. (2007). Using data mining technology to provide a recommendation service in the digital library // The Electronic Library. Emerald Group Publishing Limited. 25:6 (2007) 711-724. DOI $10.1108 / 02640470710837137$. www.emeraldinsight.com/0264-0473.htm (28 mai. 2008).

Cardoso, J. C.; Oliveira, J. B. (2000). Problemática em Interfaces de busca de bibliotecas digitais. Porto Alegre, 2000. http://lsm.dei.uc.pt/ribie/docfiles/txt200372912310 
Problem\%C3\%A1ticas\%20em\%20interfaces $\% 20$ de $\% 20$ busca.pdf (23 set. 2008).

Dias, C. (2003). Usabilidade na Web: criando portais mais acessíveis. Rio de Janeiro: Alta Books, 2003.

Eirinaki, M.; Lampos, C.; Paulakis, S.; Vazirgiannis, M. (2004). Web Personalization Integrating Content Semantics and Navigational Patterns. // ACM - WIDM '04: Proceedings of the 6th annual ACM international workshop on Web information and data management (2004) 7279. http://portal.acm.org/ft_gateway.cfm?id=1031468\&ty pe $=$ pdf \& coll=Portal\&dl=ACM\&CFID $=29687628 \& C F T O K$ $\mathrm{EN}=12483935$ (05 mai. 2008).

Herlocker, J. L.; Konstan, J. K.; Terveen, L. G.; Riedl, John, T. (2004). Evaluating Collaborative Filtering Recommender Systems. // ACM Transactions on Information Systems, 22:1(January 2004) 5-53. http://portal.. cm.org/citation.. fm $? \mathrm{id}=963772 \& \mathrm{dl}=$ portal\& $\mathrm{dl}=\mathrm{ACM}$ (21 mar. 2008).

Jeevan, V. K. J; Padhi, P. (2006). A selective review of research in content personalization. Library Review. Emerald Group Publishing Limited, 55:9 (2006) 556-586. DOI 10.1108/00242530610706761. www.emeraldinsight. com/0024-2535.htm (28 mai. 2008).

Lima-Marques, M.; Macedo, F. L. O. (2006). Arquitetura da Informação: Base para a gestão do conhecimento. // Tarapanoff, Kira (Org). Inteligência, informação e conhecimento em corporações. Brasília: IBICT, UNESCO, 2006.

Mobasher, B.; Dai, H.; Luo, T.; Nakagawa, M. (2001). Effective Personalization Based on Association Rule Discovery from Web Usage Data. ACM - Proceedings of the 3rd international workshop on Web information and data management (2001) 9-15. // http://portal.acm.org/ft gate way. $\mathrm{cfm} ? \mathrm{id}=502935 \&$ type $=$ pdf $\&$ coll $=$ Portal $\& \mathrm{dl}=\mathrm{ACM} \& \mathrm{CF}$ ID=29687628\&CFTOKEN=12483935 (05 mai. 2008).

Morville, P.; Rosenfeld, L. Information Architecture for the World Wide Web. Sebastopol, CA: O'Reilly, 2006. 504 p.

Rosenfeld, L.; Morville, P. (1998). Information Architecture for the World Wide Web. Sebastopol, CA: O'Reilly, 1998. $202 p$.

Schilke, S. W; Bleimann, U.; Furnell, S. M; Phippen, A. D.Multi-dimensionalpersonalisation for location and interestbased recommendation. // Internet Research. Emerald Group Publishing Limited. 14:5 (2004) 3793851066-2243. DOI 10.1108/10662240410566980. http://www.emeraldinsight.com/1066-2243.htm (28 mai. 2008).

Tosete Herranz, F.; Rodriguez Mateos, D. (2004). Arquitectura de la información y el diseño de sedes web. Universidad Carlos III de Madrid. // Sebastian, Mercedes Caridad; Flores, J. Tomás Nogales (coord.). La Información en la posmodernidad: la sociedad del conocimiento en España e iberoamérica. Editorial universitária Ramón Areces, 2004

Vieira, F. J. T. (2005). Personalização de informações em portais corporativos - o caso do SERPRO. Dissertação (Mestrado em Ciência da Informação). 106 p. UnB - Universidade de Brasília, Brasília - DF, 2005.

Walter, F. E.; Battiston, S.; Schweitzer, F (2007). A model of a trust-based recommendation system on a social network. Springer Science; Business Media, 16:1 (2007) 57-74. http://www.springerlink.com/content/yp94v7553p3 22072/fulltext.pdf. DOI 10.1007/s10458-007-9021-x. (05 mai. 2008). 